\title{
Intrahepatic biloma following transcatheter arterial chemoembolization for hepatocellular carcinoma: Incidence, imaging features and management
}

\author{
BO ZHANG $^{1}$, YONGJIAN GUO ${ }^{1}$, KETONG WU $^{1}$ and HONG SHAN ${ }^{2}$ \\ ${ }^{1}$ Department of Radiology, The Sixth Affiliated Hospital; ${ }^{2}$ Department of Radiology, \\ The Third Affiliated Hospital, Sun Yat-sen University, Guangzhou, Guangdong 510655, P.R. China
}

Received October 19, 2016; Accepted February 24, 2017

DOI: $10.3892 / \mathrm{mco} .2017 .1235$

\begin{abstract}
Repeat transcatheter arterial chemoembolization (TACE) becomes more challenging for patients with intrahepatic biloma following TACE for hepatocellular carcinoma (HCC).The purpose of this study was to investigate the clinical course, incidence, imaging features and outcome and to explore the reasonable therapy scheme for intrahepatic biloma following TACE for HCC.A total of 4,695 TACE procedures were performed for 1,923 patients with HCC. Twenty patients with intrahepatic biloma following TACE were studied retrospectively. The incidence of intrahepatic biloma was $1.04 \%$ in this study. The 20 patients underwent 55 TACE procedures (mean, 2.75). Portal vein invasion was found in half of the patients. Eleven patients developed round solitary or multiple cystic biloma, 6 patients had branched biloma and 3 patients developed both cystic and branched biloma. Percutaneous drainage was applied for 4 patients. One patient underwent partial hepatectomy and one mortality occurred due to progressive biloma and multiple organ failure. Although severe intrahepatic biloma following TACE is rare, the procedure should be performed with caution. Timely and appropriate management, including percutaneous drainage, partial hepatectomy and antibiotic administration should be performed in the case of any signs of infection.
\end{abstract}

\section{Introduction}

The incidence of hepatocellular carcinoma (HCC) has increased significantly over previous decades, and globally it represents the sixth most common cancer type and the third highest cause of

Correspondence to: Professor Bo Zhang, Department of Radiology, The Sixth Affiliated Hospital, Sun Yat-sen University, Guangzhou, Guangdong 510655, P.R. China

E-mail: zhangbo19790616@163.com

Key words: intrahepatic biloma, transcatheter arterial chemoembolization, hepatocellular carcinoma, percutaneous drainage cancer mortality among the general population (1). Hepatitis B and $\mathrm{C}$ and alcohol abuse are major risk factors for HCC. Although local ablation techniques and multikinase inhibitors, such as radiofrequency ablation and sorafenib, are increasingly prevalent, transcatheter arterial chemoembolization (TACE) remains one of the standard treatments for patients with intermediate- and advanced-stage HCC (1-3). Several randomized controlled studies have revealed that TACE improves survival and controls the symptoms of HCC (4-8).

Despite its significant anti-tumor effects, repeat TACE is frequently required in patients with HCC and can cause more local complications than conservative management. To minimize the risk associated with TACE, it is important to understand the major complications of this procedure. The most common and severe complication is TACE-associated hepatic and biliary damage, which primarily consists of hepatic insufficiency, liver abscess and intrahepatic biloma formation among others (9).

Once intrahepatic biloma has developed, repeat TACE becomes more difficult and riskier in patients with HCC (9). Therefore the methods for effective prevention and treatment of intrahepatic biloma after TACE must be determined. The purpose of this study was to clarify the clinical course, incidence, risk factors, interventional management and outcome of intrahepatic biloma following TACE.

\section{Patients and methods}

Patients. A total of 4,695 TACE procedures were performed for the 1,923 patients with HCC in the third affiliated hospital (between January 2007 and October 2012) and in the sixth affiliated hospital (between November 2012 and July 2015) of Sun Yat-sen University (Guangzhou, China). Of these patients, 20 consecutive cases of intrahepatic biloma following TACE confirmed by clinical history and computed tomography (CT) and/or magnetic resonance imaging (MRI) were analyzed retrospectively. The study protocol was approved by the Institutional Ethics Review Board of our hospitals. Written informed consent was obtained from each patient.

The diagnosis of HCC was established by dynamic radiological finding and clinical data. The conventional triple-phase dynamic radiological behavior of HCC included 

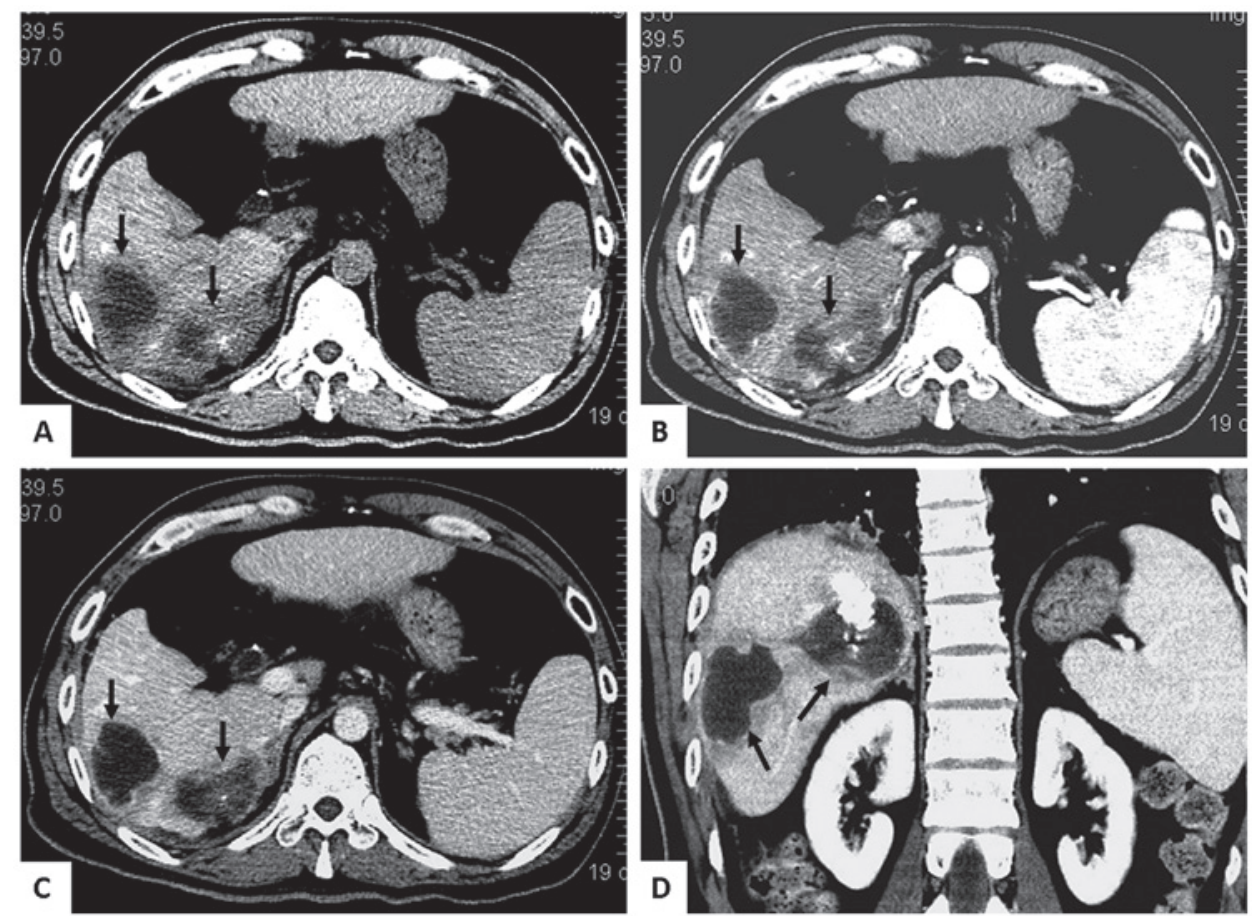

Figure 1. Multiple cystic biloma in a 50-year old man secondary to 3 transcatheter arterial chemoembolization procedures. (A-C) Axial and (D) coronal reconstruction computed tomography scans revealed multiple cystic lesions (arrow) in the nontumoral liver parenchyma without enhancement in the (B) arterial phase and (C) portal phase following injection of iodine contrast.

early enhancement on the arterial phase and fast washout on the portal venous and delayed venous phases (10). Chronic hepatitis B, chronic hepatitis $\mathrm{C}$, liver cirrhosis and tumor markers (such as $\alpha$-fetoprotein) were also considered as other supporting evidence for the presence of HCC.

According to previous reports $(9,11)$, the diagnosis of intrahepatic biloma following TACE was based on i) round, solitary or multiple cystic lesions located in nontumoral parenchyma with or without segmental bile duct dilatation or ii) a branched hypoattenuating area along the Glisson's sheath similar to dilatation of the intrahepatic bile duct without enhancement at any of the vascular phases on follow-up CT scans. On MRI scans, these lesions demonstrated hypointensity and hyperintensity on T1- and T2-weighted images, respectively, without evidence of enhancement.

Procedure. TACE was typically performed using the following steps. A right transfemoral approach was used for artery access, and following insertion of a 4- to 5-French catheter into the hepatic artery, the feeding arteries were evaluated using hepatic angiography. For the fine and tortuous feeding artery, a 2.2-2.8 French microcatheter was used to minimize the embolized area. Subsequent to microcatheter insertion into the proper branch, chemoembolization was performed through the injection of a 2-50 ml mixture of anticancer drugs and iodized oil (Lipiodol; Andre Guerbet, Aulnaysous-Bois, France) with or without gelfoam or polyvinyl alcohol particles. The total amount of mixture in a single procedure was determined based on tumor size and blood supply. No more than three of the following anticancer drugs were used for each procedure: Doxorubicin hydrochloride (10-30 mg), epirubicin hydrochloride (10-30 mg), mitomycin C (6-10 mg), nedaplatin
(40-100 mg), lobaplatin (50-100 mg), cisplatin $(25-100 \mathrm{mg})$, oxaliplatin (50-200 mg) and 5-fluorouracil (250-1250 mg).

Follow-up and data analysis. Dynamic enhanced CT or MRI was performed every 1-2 months after TACE to check for iodized oil distribution and tumor recurrence. If local recurrence or new lesions were confirmed, an additional TACE procedure and/or other treatment (e.g. radiofrequency or microwave ablation, percutaneous ethanol injection, radioactive ${ }^{125} \mathrm{I}$ seed implantation or systemic chemotherapy) were performed. Once the signs of intrahepatic biloma formation were detected, these patients were monitored closely. Percutaneous puncture into the biloma cavity directly and aspiration or drainage were performed for infectious lesions and jaundice.

Clinical data were analyzed with respect to age, gender, Child-Pugh classification (11), main tumor size, TACE procedures, volume of ipiodol administered, treatment and outcome of biloma, which were collected from the original hospital charts, operation notes and outpatient medical records via telephone questionnaires. The end-point of follow-up was the time of patient mortality and liver transplantation. Data analysis was performed using SPSS version 19.0 (IBM SPSS, Armonk, NY, USA) to generate Kaplan-Meier curves.

\section{Results}

Baseline characteristics. As listed in Table I, a total of 20 patients were included for analysis. There were 19 males and 1 female (mean age, 51 years; range, 20-76 years). The incidence of intrahepatic biloma following TACE was $1.04 \%$ in this series. Prior to intrahepatic biloma formation, the 20 patients underwent 55 TACE procedures (mean per 


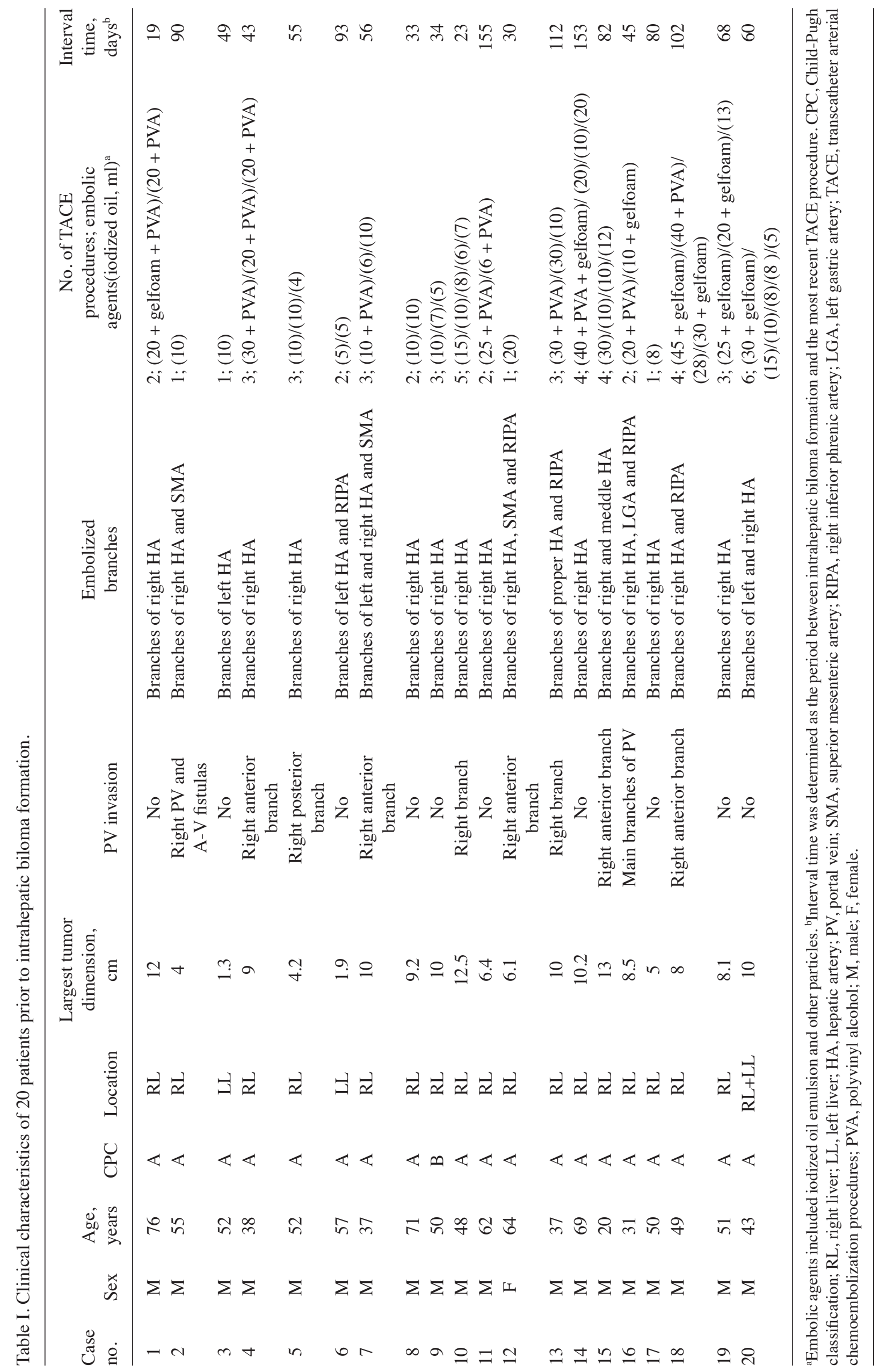




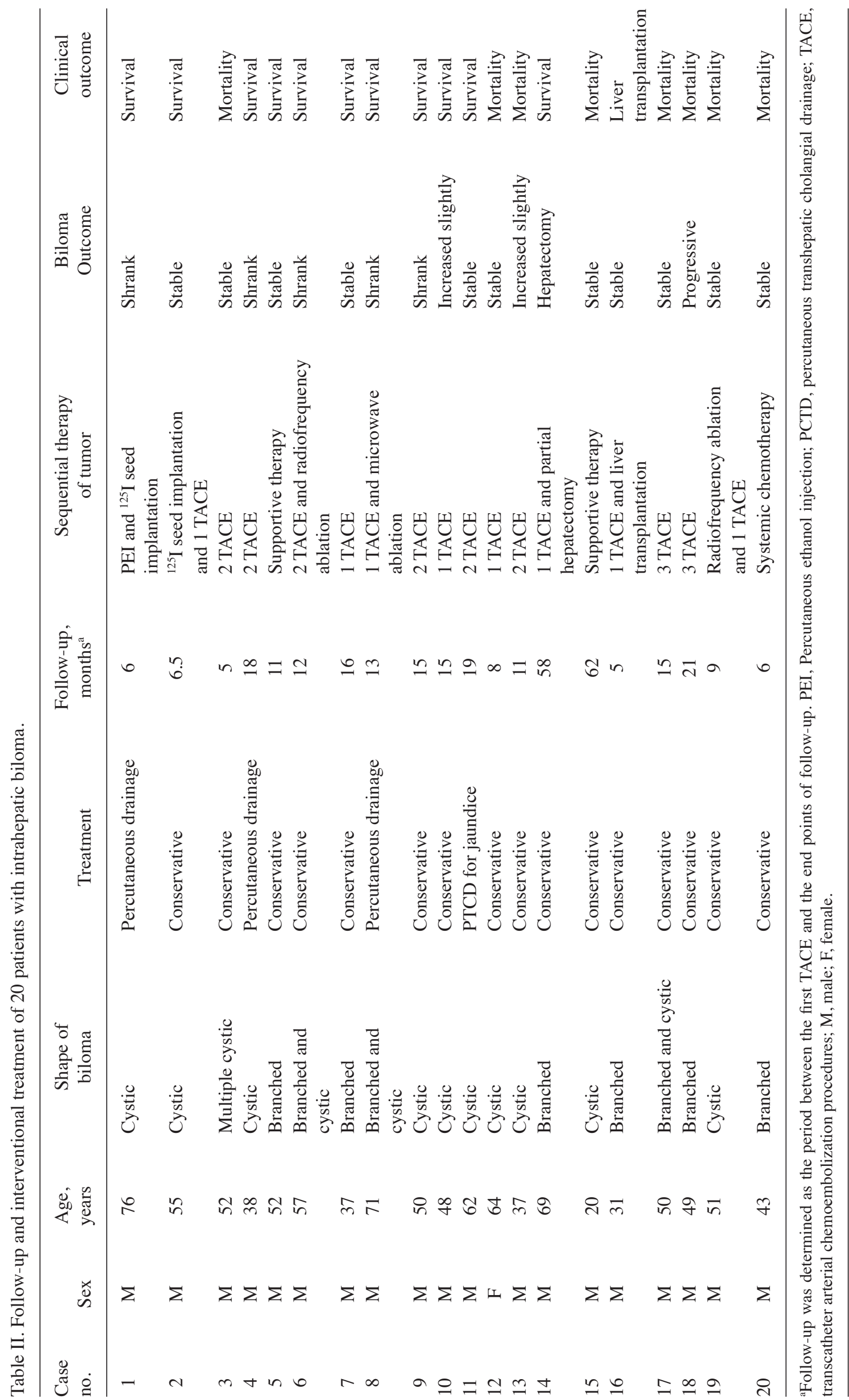



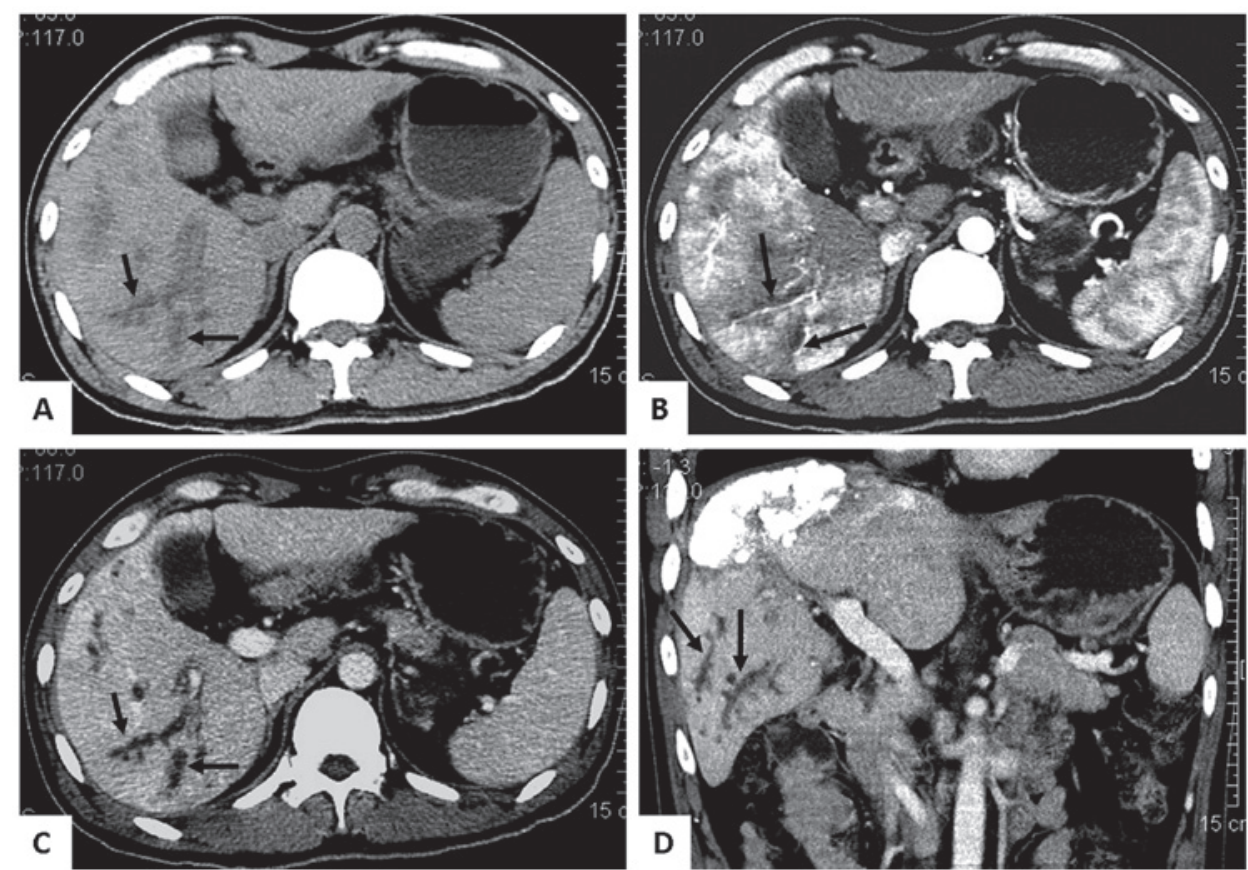

Figure 2. Branched biloma in a 37-year old man following 3 transcatheter arterial chemoembolization procedures. (A-C) Axial and (D) coronal reconstruction computed tomography images revealing a branched hypoattenuating area (arrow) along the Glisson's sheath, mimicking dilatation of the intrahepatic bile duct without enhancement in the (B) arterial phase and (C) portal phase.

patient, 2.75; range, 1-6) and the mean HCC diameter was $7.97 \mathrm{~cm}$ (range, $1.3-13 \mathrm{~cm}$ ). The rate of portal vein invasion was $50 \%(10 / 20)$. The mean interval time between intrahepatic biloma formation and most recent TACE procedure was 69.1 days (range, 19-155 days).

As presented in Table II, eleven patients developed round solitary or multiple cystic biloma (Fig. 1), six patients developed branched biloma (Fig. 2) mimicking diffuse bile duct dilatation and the remaining 3 patients had cystic and branched biloma.

Interventional treatment of the biloma. Four patients with infection symptoms (jaundice, upper abdominal pain and fever) were treated through percutaneous transhepatic cholangial drainage (PTCD, $n=1)$ and percutaneous drainage $(n=3)$ under ultrasonography or CT guidance.

Conservative medical treatment was performed in the remaining 16 patients. During follow-up, the size of the biloma was reduced, remained stable or slightly increased in 2, 10 and 2 patients, respectively. One patient underwent partial hepatectomy for jaundice and the increase of biloma. The other patient succumbed to progressive biloma and multiple organ failure.

Intrahepatic biloma formation made the repeat TACE more challenging and riskier to perform in these patients. On average, only 1 TACE procedure (range 0-3 procedures) was performed in this group, and the sequential therapy included radioactive ${ }^{125}$ I seed implantation $(n=2)$, microwave ablation $(n=1)$, radiofrequency ablation $(n=2)$, percutaneous ethanol injection $(n=1)$, partial hepatectomy $(n=1)$, systemic chemotherapy $(n=1)$, supportive therapy $(\mathrm{n}=2)$ and liver transplantation $(\mathrm{n}=1)$.

Survival analysis. Kaplan-Meier curves revealed the cumulative survival rate of intrahepatic biloma patients following TACE

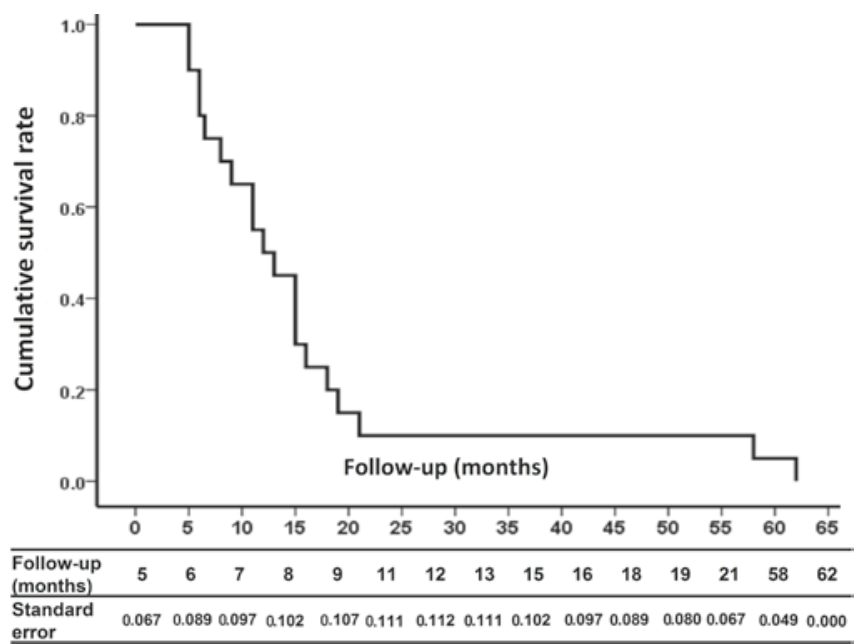

Figure 3. Kaplan-Meier curve revealing the cumulative survival rate of intrahepatic biloma patients following transcatheter arterial chemoembolization procedure for hepatocellular carcinoma. The standard error of the mean exceeded $10 \%$ from 8 to 15 months.

for HCC (Fig. 3). The standard error of the mean exceeded $10 \%$ from 8-15 months after formation of intrahepatic biloma.

\section{Discussion}

Intrahepatic biloma may easily be confirmed using enhanced CT or MRI. The incidence of intrahepatic biloma following TACE varies greatly from $0.05 \%$ (12) to $1.04 \%$ (in the present study) to $11.3 \%$ (13). The incidence discrepancy between the present study and previous reports may be partially associated with the patient population, embolic material, anticancer drugs and the TACE procedure. 
The liver is dually supplied by hepatic arterial and portal venous blood; however, HCC is primary supplied by the former. Therefore, TACE preferentially interrupts the blood supply of HCC cells and controls tumor growth. Unlike the normal liver parenchyma, the intrahepatic bile ducts are supplied exclusively by the hepatic arterial branches that form a vascular plexus (peribiliary capillary plexus) around the bile ducts. During the TACE procedure, the peribiliary capillary plexus is filled with iodized oil or other embolic material. Therefore, ischemia of the intrahepatic bile ducts may easily occur following TACE (13-15).

Liver cirrhosis has a notable role in the formation of intrahepatic biloma following TACE. In the cirrhotic liver, hypertrophy of the peribiliary capillary plexus may function as a portoarterial shunt and compensate for the occluded arterial flow; therefore, the hypertrophied peribiliary capillary plexus in the cirrhotic liver is able to prevent the ischemic injury of bile ducts during TACE $(13,16,17)$. On the other hand, the high incidence of bile duct injury in patients with metastatic tumors and normal liver morphology also indicates the protective role of the hypertrophied peribiliary plexus around the bile ducts $(9,18)$. The low incidence $(1.04 \%)$ in this study may be explained as the majority of patients had HCC originating from the cirrhotic liver with hepatitis B virus infection.

The other risk factors for intrahepatic biloma may include portal vein thrombosis, biliary obstruction and inflammation, the total number of TACE procedures undergone, and the total volume of iodized oil, anticancer drugs, microspheres, gelfoam and drug-eluting beads administered and undergoing segmental or subsegmental TACE $(13,18-21)$. However, in the present study, with the exception of portal vein invasion in half of the patients, there was no obvious trend of repeat TACE procedures, embolic materials and anticancer drugs. Potentially due to the low incidence and lack of prospective studies of intrahepatic biloma following TACE, these risk factors are not widely recognized.

In the majority of cases, intrahepatic biloma may be treated conservatively. However, for moderate to severe signs of infection with or without increases in the size of the biloma, promptly percutaneous drainage or partial hepatectomy even internal drainage combined with antibiotics should be applied first (9,22-24). If it is not treated in a timely and appropriate manner, it may result in severe systemic infection, and even a bronchobiliary fistula or biliopleural fistula and multiple organ failure $(23,25)$. In the current series, one patient succumbed to progressive biloma and septic shock.

Repeat TACE becomes more challenging and is associated with increased risk in patients with intrahepatic biloma. On average, only 1 TACE (range, 0-3) procedure per patient was performed in this series after formation of intrahepatic biloma, and the sequential therapy included local treatment (radioactive ${ }^{125} \mathrm{I}$, microwave or radiofrequency ablation, percutaneous ethanol injection, partial hepatectomy), systemic chemotherapy (folinic acid, 5-Fluorouracil and oxaliplatin, sorafenib), supportive therapy and liver transplantation.

In conclusion, whilst severe intrahepatic biloma following TACE is rare, the procedure must be performed cautiously with superselection of the hepatic artery. If intrahepatic biloma occurs, careful observations must be made during follow-up. Timely and appropriate management including percutaneous drainage, partial hepatectomy and antibiotic administration must be performed in the case of signs of infection.

\section{Acknowledgments}

This study was supported by a grant from National Natural Science Foundation of China (grant no. 81301978), Ph.D. Programs Foundation of Ministry of Education of China (grant no. 20130171120105), Young Teacher Cultivation Project of Sun Yat-sen University (grant no. 13YKPY39) and Guangdong Province Medical Science Foundation (grant no. B2013162).

\section{References}

1. Lencioni R and Crocetti L: Local-regional treatment of hepatocellular carcinoma. Radiology 262: 43-58, 2012.

2. Llovet JM and Bruix J: Systematic review of randomized trials for unresectable hepatocellular carcinoma: Chemoembolization improves survival. Hepatology 37: 429-442, 2003.

3. Xue TC, Xie XY, Zhang L, Yin X, Zhang BH and Ren ZG: Transarterial chemoembolization for hepatocellular carcinoma with portal vein tumor thrombus: A meta-analysis. BMC Gastroenterol 13: 60, 2013.

4. Lo CM, Ngan H, Tso WK, Liu CL, Lam CM, Poon RT, Fan ST and Wong J: Randomized controlled trial of transarterial lipiodol chemoembolization for unresectable hepatocellular carcinoma. Hepatology 35: 1164-1171, 2002.

5. Lo GH: The use of transarterial chemoembolization in hepatiocellular carcinoma $2 \mathrm{~cm}$ or smaller. Am J Gastroenterol 110: 196, 2015.

6. Chern MC, Chuang VP, Liang CT, Lin ZH and Kuo TM: Transcatheter arterial chemoembolization for advanced hepatocellular carcinoma with portal vein invasion: Safety, efficacy, and prognostic factors. J Vasc Interv Radiol 25: 32-40, 2014.

7. Llovet JM, Real MI, Montaña X, Planas R, Coll S, Aponte J, Ayuso C, Sala M, Muchart J, Solà R, et al: Arterial embolisation or chemoembolisation versus symptomatic treatment in patients with unresectable hepatocellular carcinoma: A randomised controlled trial. Lancet 359: 1734-1739, 2002.

8. Lewandowski RJ, Mulcahy MF, Kulik LM, Riaz A, Ryu RK, Baker TB, Ibrahim SM, Abecassis MI, Miller FH, Sato KT, et al: Chemoembolization for hepatocellular carcinoma: Comprehensive imaging and survival analysis in a 172-patient cohort. Radiology 255: 955-965, 2010.

9. Sakamoto I, Iwanaga S, Nagaoki K, Matsuoka Y, Ashizawa K, Uetani M, Fukuda T, Okimoto T, Okudaira S, Omagari K, et al: Intrahepatic biloma formation (bile duct necrosis) after transcatheter arterial chemoembolization. AJR Am J Roentgenol 181: 79-87, 2003.

10. Iavarone M, Sangiovanni A, Forzenigo LV, Massironi S, Fraquelli M, Aghemo A, Ronchi G, Biondetti P, Roncalli M and Colombo M: Diagnosis of hepatocellular carcinoma in cirrhosis by dynamic contrast imaging: The importance of tumor cell differentiation. Hepatology 52: 1723-1730, 2010.

11. Guiu B, Deschamps F, Aho S, Munck F, Dromain C, Boige V, Malka D, Leboulleux S, Ducreux M, Schlumberger M, et al: Liver/biliary injuries following chemoembolisation of endocrine tumours and hepatocellular carcinoma: Lipiodol vs. drug-eluting beads. J Hepatol 56: 609-617, 2012.

12. Xia J, Ren Z, Ye S, Sharma D, Lin Z, Gan Y, Chen Y, Ge N, Ma Z, Wu Z, et al: Study of severe and rare complications of transarterial chemoembolization (TACE) for liver cancer. Eur J Radiol 59: 407-412, 2006.

13. Yu JS, Kim KW, Jeong MG, Lee DH, Park MS and Yoon SW: Predisposing factors of bile duct injury after transcatheter arterial chemoembolization (TACE) for hepatic malignancy. Cardiovasc Intervent Radiol 25: 270-274, 2002.

14. Chung J, Yu JS, Chung JJ, Kim JH and Kim KW: Haemodynamic events and localised parenchymal changes following transcatheter arterial chemoembolisation for hepatic malignancy: Interpretation of imaging findings. Br J Radiol 83: 71-81, 2010.

15. Spina JC, Ulla M, Yeyati EL, Kucharczyk MC, Irusta H, Savluk JL and García-Mónaco R: MDCT findings after hepatic chemoembolization with DC-beads: What the radiologist needs to know. Abdom Imaging 38: 778-784, 2013. 
16. Demachi H, Matsui O, Kawamori Y, Ueda K and Takashima T: The protective effect of portoarterial shunts after experimental hepatic artery embolization in rats with liver cirrhosis. Cardiovasc Intervent Radiol 18: 97-101, 1995.

17. Zipprich A, Loureiro-Silva MR, D'Silva I and Groszmann RJ: The role of hepatic arterial flow on portal venous and hepatic venous wedged pressure in the isolated perfused CCl4-cirrhotic liver. Am J Physiol Gastrointest Liver Physiol 295: G197-G202, 2008.

18. Bhagat N, Reyes DK, Lin M, Kamel I, Pawlik TM, Frangakis C and Geschwind JF: Phase II study of chemoembolization with drug-eluting beads in patients with hepatic neuroendocrine metastases: High incidence of biliary injury. Cardiovasc Intervent Radiol 36: 449-459, 2013.

19. Chen MJ, Lin CC, Chang WH and Yang FS: Biloma following repeated transcatheter arterial embolization and complicated by intrahepatic duct stones: A case report. World J Gastroenterol 11: 4764-4765, 2005.

20. Chung JW, Park JH, Han JK, Choi BI, Han MC, Lee HS and Kim CY: Hepatic tumors: Predisposing factors for complications of transcatheter oily chemoembolization. Radiology 198: 33-40, 1996.

21. Naumann M, Bonsall R and Gupta R: Chemoembolization with drug-eluting beads complicated by intrahepatic biloma. Semin Intervent Radiol 28: 212-217, 2011
22. Maruyama T, Mori A, Tatebe H, Sakai K, Isono N, Ohashi N, Inoue $\mathrm{H}$, Takegoshi $\mathrm{S}$ and Okuno M: A novel technique for the internal drainage of extrahepatic biloma complicating transarterial embolization of hepatocellular carcinoma. J Gastroenterol 42: 783-786, 2007.

23. Akazawa S, Omagari K, Amenomori M, Nishiyama H, Mizuta Y and Kohno S: Bronchobiliary fistula associated with intrahepatic biloma after transcatheter arterial chemoembolization for hepatocellular carcinoma. J Hepatol 40: 1045-1046, 2004.

24. Sun Z, Li G, Ai X, Luo B, Wen Y, Zhao Z, Dong S and Guan J: Hepatic and biliary damage after transarterial chemoembolization for malignant hepatic tumors: Incidence, diagnosis, treatment, outcome and mechanism. Crit Rev Oncol Hematol 79: 164-174, 2011.

25. Butt AS, Mujtaba G, Anand S and Krishnaiah M: Management of biliopleural fistula after transarterial chemoembolization of a liver lesion. Can J Gastroenterol 24: 281-283, 2010. 